ARTICLE

\title{
Combining high-resolution scanning tunnelling microscopy and first-principles simulations to identify halogen bonding
}

James Lawrence (10 ${ }^{1}$, Gabriele C. Sosso (10) ${ }^{1,2 凶}$, Luka Đorđević (iD ${ }^{3}$, Harry Pinfold ${ }^{1}$, Davide Bonifazi (i) ${ }^{3 凶} \&$ Giovanni Costantini (iD ${ }^{1 \times}$

Scanning tunnelling microscopy (STM) is commonly used to identify on-surface molecular self-assembled structures. However, its limited ability to reveal only the overall shape of molecules and their relative positions is not always enough to fully solve a supramolecular structure. Here, we analyse the assembly of a brominated polycyclic aromatic molecule on $\mathrm{Au}(111)$ and demonstrate that standard STM measurements cannot conclusively establish the nature of the intermolecular interactions. By performing high-resolution STM with a COfunctionalised tip, we clearly identify the location of rings and halogen atoms, determining that halogen bonding governs the assemblies. This is supported by density functional theory calculations that predict a stronger interaction energy for halogen rather than hydrogen bonding and by an electron density topology analysis that identifies characteristic features of halogen bonding. A similar approach should be able to solve many complex 2D supramolecular structures, and we predict its increasing use in molecular nanoscience at surfaces.

\footnotetext{
${ }^{1}$ Department of Chemistry, University of Warwick, Gibbet Hill Road, Coventry CV4 7AL, UK. ${ }^{2}$ Centre for Scientific Computing, University of Warwick, Gibbet Hill Road, Coventry CV4 7AL, UK. ${ }^{3}$ School of Chemistry, Cardiff University, Park Place Main Building, Cardiff CF10 3AT, UK. ${ }^{\circledR}$ email: G.Sosso@warwick.ac.uk, BonifaziD@cardiff.ac.uk; G.Costantini@warwick.ac.uk
} 
$\mathrm{n}$ order to engineer robust functional molecular nanostructures at surfaces, self-assembly via strong, directional intermolecular forces is often required. Both halogen ${ }^{1}$ and hydrogen bonding ${ }^{2}$ possess these characteristics to different extents, offering different potential nanofabrication pathways and allowing for a diverse set of molecular moieties that can participate in the formation of supramolecular architectures. There are many examples in the literature of two-dimensional (2D) hydrogen bonded $(\mathrm{HB})$ assemblies on surfaces that have been examined with surface science techniques ${ }^{3-6}$. Halogen-bonded (XB) molecular nanostructures ${ }^{1,7-9}$ are considerably less common in on-surface $2 \mathrm{D}$ systems but are increasingly being studied and seen as an important addition to the 'toolbox' of supramolecular assembly. Typically, iodine $\mathrm{e}^{10-14}$ and bromine atoms $\mathrm{s}^{15-19}$ are used as halogen bond donors due to their strong polarisability, but examples of chlorine $^{20}$ and fluorine $\mathrm{e}^{21,22}$ based assemblies have also been reported. Halogen bonds can also involve halogen atoms acting simultaneously as both donor and acceptor ${ }^{11,16,17,23,24}$, with the positive electrostatic potential of the sigma hole oriented towards the central 'belt' of negative electrostatic potential found on an adjacent halogen atom. Alternatively, nitrogen $10,12,13,19$ and oxygen ${ }^{17,25-27}$ atoms act as acceptor sites in heteromolecular assemblies.

2D assemblies are commonly characterised on surfaces with scanning probe microscopy (SPM) techniques such as scanning tunnelling microscopy (STM) and atomic force microscopy (AFM). In recent years, higher resolution forms of SPM have become available $\mathrm{e}^{28-32}$ that make use of functionalised tips (such as $\mathrm{CO}, \mathrm{Xe}, \mathrm{D}_{2}, \mathrm{H}_{2}, \mathrm{Br}$, and $\left.\mathrm{CuO}\right)^{28,30,31,33,34}$ to reveal the internal structure of molecules adsorbed on surfaces with astounding clarity $^{29}$. Non-contact AFM (NC-AFM) and high-resolution STM (HR-STM) have been used to resolve the structures of molecules that are difficult to determine with more traditional analytical methods such as NMR or mass spectrometry ${ }^{35-37}$, as well as to identify intermediates and products of reactions that have taken place on surfaces ${ }^{38-41}$. In particular, NC-AFM and HR-STM have been employed for examining the internal structure of graphene nanostructures, as the number and type of molecular rings can easily be resolved ${ }^{42-45}$. There are also various examples of the use of these techniques for studying the supramolecular structure of $2 \mathrm{D}$ self-assembled layers ${ }^{21,46-49}$, although in most of these cases the chemical structure of the molecular components led to an unambiguous assignment of the type of intermolecular bonding.

Here we identify a case-the self-assembly of 3,9-dibromoperi-xanthenoxanthene $\left(3,9-\mathrm{Br}_{2} \mathrm{PXX}\right)$ molecules on a $\mathrm{Au}(111)$ surface-where the potential coexistence of different intermolecular interactions presents a formidable challenge for standard forms of SPM. In particular, while conventional STM measurements cannot determine whether 3,9- $\mathrm{Br}_{2} \mathrm{PXX}$ assembles via $\mathrm{HB}$ or $\mathrm{XB}$, only by means of HR-STM are we able to identify $\mathrm{XB}$ interactions as the driving force leading to the formation of supramolecular assemblies of $3,9-\mathrm{Br}_{2} \mathrm{PXX}$ on $\mathrm{Au}(111)$.

\section{Results}

Molecular assembly. 3,9- $\mathrm{Br}_{2} \mathrm{PXX}$ (Fig. 1a) is a dibrominated derivative of PXX, an electron donor molecule ${ }^{50-53}$, derivatives of which have previously been employed as $p$-type semiconductors in organic thin-film transistors due to their efficient carrier injection properties, high mobility, and thermal stability ${ }^{54,55}$. The $\mathrm{Br}$ and $\mathrm{O}$ atoms in $3,9-\mathrm{Br}_{2} \mathrm{PXX}$ offer the possibility of both $\mathrm{HB}$ and $\mathrm{XB}$ intermolecular interactions when arranged into supramolecular arrays on a surface. $\mathrm{HB}$ can originate from nonclassical weak $\mathrm{C}-\mathrm{H} \cdots \mathrm{O}$ interactions, while the possibility of $\mathrm{XB}$ stems from the emergence of the so-called $\sigma$-hole ${ }^{56}$ on the $\mathrm{Br}$ atom (Fig. 1b). Density functional theory (DFT) calculations
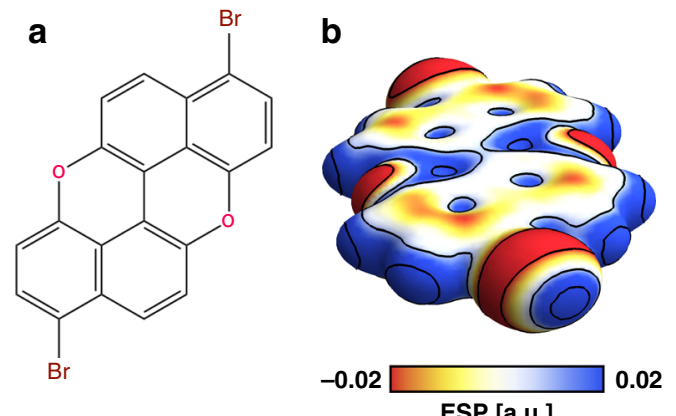

Fig. 1 Structure and electrostatic potential of $3,9-\mathbf{B r}_{2} \mathbf{P X X}$. a Molecular structure of $3,9-\mathrm{Br}_{2} \mathrm{PXX}$. $\mathbf{b}$ Map of the electrostatic potential (ESP) projected on an isosurface (0.001 a.u.) of the electron density, clearly showing the $\sigma$ hole and the corresponding electron-rich belt on the $\mathrm{Br}$ atoms. Continuous lines separate ESP regions differing by more than 0.01 a.u.

(see Methods) show that there is a region of negative electrostatic potential that forms a belt around the $\mathrm{C}-\mathrm{Br}$ bonds, while a region of positive potential (the $\sigma$-hole) develops in the elongation of the same bond, thus providing the opportunity for $\mathrm{Br}$ and $\mathrm{O}$ atoms to act as halogen bond donors and acceptors, respectively.

At low coverages, the majority of the $3,9-\mathrm{Br}_{2} \mathrm{PXX}$ molecules self-assemble into kagome-type structures (phase 1) that develop in the face-centred cubic ( $\mathrm{fcc}$ ) regions and elbow sites of the $\mathrm{Au}$ (111) herringbone reconstruction (Fig. 2a). At $77 \mathrm{~K}$, the peripheries of the kagome islands are often seen to vary between scans, indicating a significant level of molecular mobility. Combined with the lack of any noticeable herringbone distortion, we attribute this to the $3,9-\mathrm{Br}_{2} \mathrm{PXX}$ molecules being weakly bound to the $\mathrm{Au}(111)$ surface (typically, strongly bound adsorbates result in a lifting or a significant distortion of the herringbone reconstruction ${ }^{57-60}$ ). Two mirror symmetric orientations of the kagome structures can be found, suggesting that the pro-chiral 3,9- $\mathrm{Br}_{2} \mathrm{PXX}$ molecules segregate into chiral domains upon adsorption. The kagome structure itself can be thought of as being composed of triangular sub-units that consist of three molecules, each appearing to be bound with the end of one molecule pointing to the side of another (Fig. 2d). While this indicates that the oxygen atoms of $3,9-\mathrm{Br}_{2} \mathrm{PXX}$ are involved in intermolecular bonding, the nature of the interaction is not clear, as is discussed below. The triangular sub-units are packed into a hexagonal unit cell with lattice vectors $\mathbf{a}=\mathbf{b}=2.2 \pm 0.1 \mathrm{~nm}$, and an angle of $60 \pm 2^{\circ}$. Full unit cells are rarely found due to the confinement of the assembly in the fcc regions of the herringbone reconstruction.

Coexisting with the kagome structure, a second minority assembly (phase 2, Fig. 2b) is observed at low molecular coverage. Phase 2 consists of irregular structures that, similar to the kagome assembly, are limited to the fcc regions of the $\mathrm{Au}(111)$ herringbone reconstruction. Within these irregular islands, variations in molecular shape are observed, with some of the ends of the molecules appearing differently, and some molecules having an extra feature at the sides (Fig. 2e). Increasing the coverage results in the development of a further, denser assembly (phase 3, Fig. 2c), where the molecules are arranged into parallel rows (Fig. 2f). This structure forms extended and compact islands that span both fcc and bcc regions of the substrate and coexist with few remaining phase 1 and phase 2 regions (Supplementary Fig. 1).

Initial modelling of the kagome assembly by overlaying molecular structures onto the STM images results in two distinct possibilities for the type of intermolecular interaction that governs the assembly. The unit cell dimensions and possible 

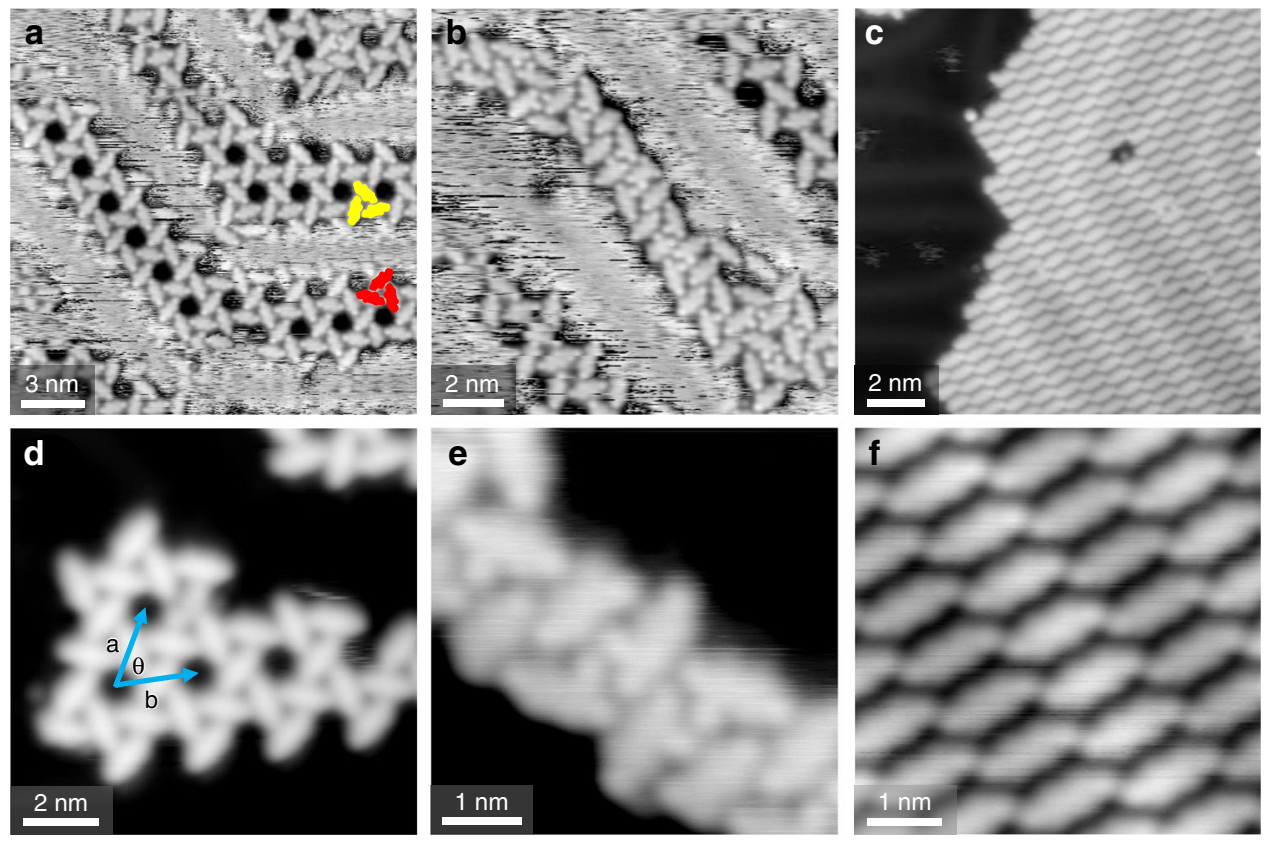

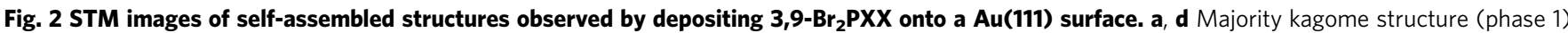
formed at low molecular coverage. Domains of opposite chirality are indicated by red/yellow highlights in $\mathbf{a}$ and the parameters of the surface unit cell are shown in d. b, e Minority irregular assembly (phase 2) coexisting with the kagome structure at low molecular coverage. c, f Compact assembly (phase 3) that develops at higher molecular coverage. STM images $\mathbf{a}$ and $\mathbf{b}$ were acquired at $77 \mathrm{~K}$, $\mathbf{c}-\mathbf{f}$ at $7 \mathrm{~K}$.
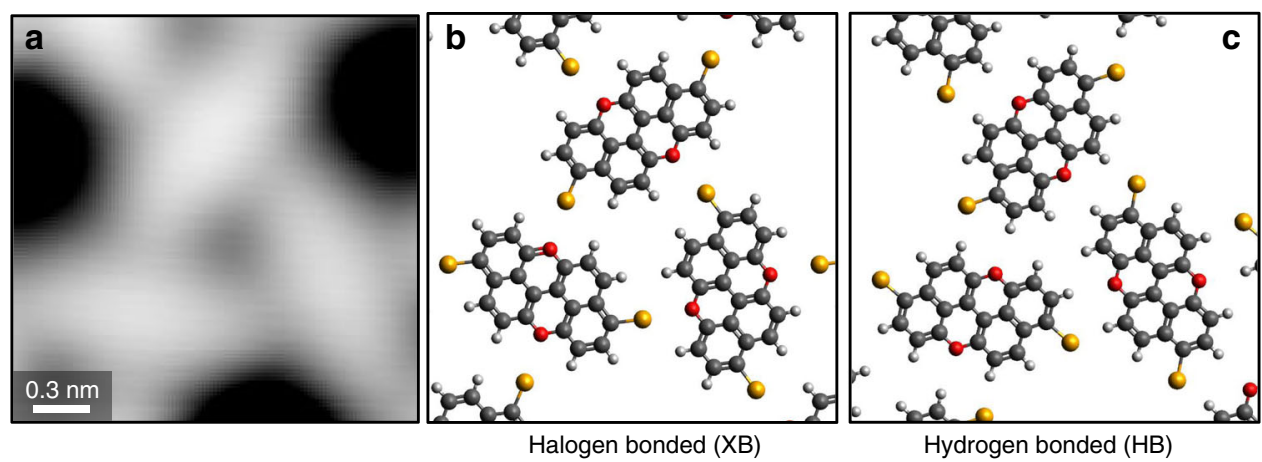

Fig. 3 Possible intermolecular bonding motifs stabilizing the kagome structure. a High magnification STM image of the kagome structure highlighting one of its composing triangular sub-units. $\mathbf{b}$ Possible halogen bonded and $\mathbf{c}$ hydrogen-bonded structures for the kagome packing.

orientations of the molecule are compatible with either halogen bonding between the $\mathrm{Br}$ end groups and the $\mathrm{O}$ atoms of adjacent molecules (Fig. 3b), or a non-classical O...H-C hydrogen bond and, possibly, a secondary electrostatic Br...H interaction (Fig. 3c). The relatively featureless appearance of the molecules in typical STM tunnelling conditions does not present an obvious solution to this, even when varying the bias voltage. In this case, the position of the molecular groups (and thus the type of assembly) can therefore not be directly inferred by using standard STM techniques.

High-resolution STM imaging. In order to clarify the structure of the kagome assembly, we harnessed the capabilities of HRSTM, which we performed at $7 \mathrm{~K}$ using a $\mathrm{CO}$-functionalised tip. In agreement with previous HR-STM experiments and theoretical studies ${ }^{42,61}$, intramolecular features could be resolved when approaching the $\mathrm{CO}$ tip close to adsorbed molecules (Fig. 4). The rings of the molecule (in particular, the naphthalene aromatic sections) are clearly resolved, as are the positions of the $\mathrm{C}-\mathrm{Br}$ end groups. This allows us to overlay a molecular model of 3,9-
$\mathrm{Br}_{2} \mathrm{PXX}$ in a single orientation for all molecules in the kagome structures of a given chirality (the flipped orientation of 3,9$\mathrm{Br}_{2} \mathrm{PXX}$ matches the HR-STM images of molecules in the kagome structures of the opposite chirality). By doing so, it appears evident that in the triangular sub-units of the kagome packing the $\mathrm{Br}$ atoms of one molecule directly face the $\mathrm{O}$ atoms of a neighbouring one, as would be expected in XB (Figs. $4 \mathrm{~d}$ and $3 \mathrm{~b}$ ). Moreover, overlaying scaled molecular models gives an estimated $\mathrm{O} \cdots \mathrm{Br}$ distance of $3.1 \pm 0.1 \AA$ and a $\mathrm{C}-\mathrm{O} \cdots \mathrm{Br}$ angle of $176 \pm 2^{\circ}$. The former is smaller than the sum of the van der Waals radii of the two elements $(3.37 \AA)^{62}$ while the latter is very close to $180^{\circ}$, thus satisfying two established criteria ${ }^{63}$ for identifying halogen bonds. All examples of the kagome packing studied by HR-STM were determined to be governed by halogen-bonding interactions. No examples of hydrogen-bonded assemblies could be found, demonstrating that the $3,9-\mathrm{Br}_{2} \mathrm{PXX}$ molecules on $\mathrm{Au}(111)$ form only XB at low coverages.

DFT calculations and image simulations. To get further insight into the nature of the intermolecular bonding, $\mathrm{XB}$ and 

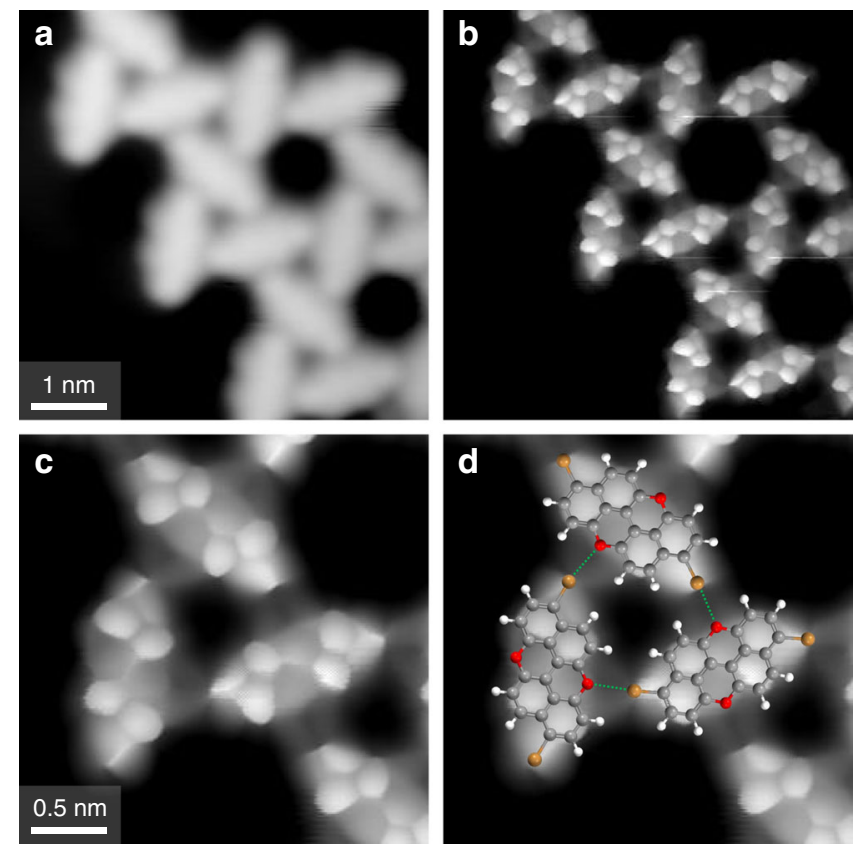

Fig. 4 High-resolution STM images of the $3,9-\mathrm{Br}_{2} \mathrm{PXX}$ kagome assembly. a Constant current STM image recorded with typical scanning parameters with a CO tip ( $V=0.51 \mathrm{~V}, I=160 \mathrm{pA})$. b Constant height HR-STM image of the same region as $\mathbf{a}(V=30 \mathrm{mV})$ with the $C O$ tip close to the surface. c Smaller scale zoom of the same area seen in b, $V=30 \mathrm{mV}$. d Molecular model overlay of $\mathbf{c}$, with halogen bonds indicated by green dotted lines. All images were recorded at $7 \mathrm{~K}$.

(hypothetical) $\mathrm{HB} 3,9-\mathrm{Br}_{2} \mathrm{PXX}$ assemblies were investigated by means of DFT calculations. As the molecules appeared to be weakly adsorbed on the relatively inert $\mathrm{Au}(111)$ surface, and since the commensurability of the molecular adlayer could not be determined from the STM measurements, the calculations were performed on free-standing monolayers, taken as reasonable theoretical approximations of the experimental structure. Similar unit cells to those observed experimentally were obtained upon the optimisation of both cell vectors and atomic positions (Supplementary Table 1). Interestingly, the average $\mathrm{O} \cdots \mathrm{H}$ distance obtained for the $\mathrm{HB}$ assembly is very similar to the $\mathrm{O} \cdots \mathrm{Br}$ distance expected for the $\mathrm{XB}$ assembly, and both are compatible with the experimentally determined value $(3.1 \pm 0.1 \AA)$. However, the XB appears to be energetically more favourable than the HB assembly by $\sim 80-100 \mathrm{meV} /$ unit cell (the precise value depending on the exchange-correlation functional used, see Supplementary Discussion). This energy difference is indicative that, in agreement with the HR-STM result, the DFT calculations predict that 3,9$\mathrm{Br}_{2} \mathrm{PXX}$ assemblies are held together preferentially by XB interactions as opposed to $\mathrm{HB}$. We argue that the preference for the $\mathrm{XB}$ network is due to the strength of the $\mathrm{O} \cdots \mathrm{Br}$ halogen bonds between 3,9- $\mathrm{Br}_{2} \mathrm{PXX}$ molecules: evaluating the "binding" energy of a 3,9-Br ${ }_{2} \mathrm{PXX}$ dimer, held together by either XB or HB interactions, results in the former being up to $\sim 25 \mathrm{meV}$ stronger than HB (see Supplementary Discussion).

It is however interesting to compare the results for the $\mathrm{XB}$ assembly with those of a hypothetical HB assembly: in Fig. 5 we report the simulated HR-STM images of both assemblies, obtained thanks to the PP-AFM/STM framework of Hapala et al. ${ }^{64}$ and Krejčí et al. ${ }^{61}$ (see Methods). The agreement between experimental (Fig. 5a) and simulated (Fig. 5b) HRSTM images of the $\mathrm{XB}$ assembly is remarkable, further supporting our assignment. On the contrary, the simulated HR-STM image of the (hypothetical) HB assembly (Fig. 5c) not only displays the wrong symmetry with respect to the experimentally observed assembly (Supplementary Fig. 6) but also suggests that the position of the atoms in the HB assembly would produce markedly different features in the HR-STM images. In particular, the bonding region in the XB assembly is characterised by two brighter triangular features crossed by dark streaks (Fig. 5b), while the HB assembly would have an overall darker appearance in (Fig. 5 c). We note that the darker streaks observed between the bromine and oxygen atoms of adjacent molecules (Figs. $4 \mathrm{c}$ and $5 \mathrm{~b}$ ) should not be interpreted as the 'imaging' of the halogen bonds, as it has been demonstrated that such intermolecular features can result from the relaxation of the flexible CO probe ${ }^{61,64}$.

Analysis of the electron density topology. The DFT calculations allows us also to investigate the electron density topology which, in conjunction with the structural features discussed earlier, provides an additional probe to determine the presence of an intermolecular XB. To this aim, we have examined the electron density $\rho$ in the proximity of the $\mathrm{O}$ and $\mathrm{Br}$ atoms, which is plotted in Fig. $6 \mathrm{a}$ as a contour map projected onto the molecular plane. It can be clearly recognised that a gradient path connects $\mathrm{O}$ and $\mathrm{Br}$ and that a saddle point, corresponding to the bond critical point (BCP), is located in between $\mathrm{O}$ and Br. This is another determining feature listed in the IUPAC definition of halogen bond ${ }^{63}$. Moreover, Fig. $6 \mathrm{~b}$ shows the computed bonding charge density difference, i.e. the electron density difference $\Delta \rho=\rho_{d}-\rho_{m 1}-$ $\rho_{m 2}$, where $\rho_{d}$ and $\rho_{m 1,2}$ refer to the electron density of the dimer and the monomers, respectively. One can notice the emergence of some charge transfer from the $\mathrm{Br}$ atom to the $\mathrm{O}$ atom, with a net accumulation of electron density (red regions) along the direction of the $\mathrm{O} \cdots \mathrm{Br}$ bond path. Also this is in agreement with the fact that "the forces involved in the formation of the halogen bond are primarily electrostatic, but polarisation, charge transfer, and dispersion contributions all play an important role" 63 . We finally note that the charge density redistribution is not limited to the close proximity of the $\mathrm{O}$ and $\mathrm{Br}$ atoms but is characterised by a rather complex pattern as could have been expected from the highly non-uniform molecular electrostatic potential in Fig. 1b. This results in the formation of a sort of binding pocket rather than a single point contact.

Identification of synthetic impurities. HR-STM also helps to elucidate the nature of the minority phase 2 by revealing the internal structure of the molecules with an "unusual" appearance and demonstrating that these differ in their configuration of $\mathrm{C}-\mathrm{Br}$ bonds from the expected $3,9-\mathrm{Br}_{2} \mathrm{PXX}$ structure. In particular, Supplementary Fig. 2 shows that some of these molecules have one terminal $\mathrm{C}-\mathrm{Br}$ group shifted by one position (i.e., they are 2,9- and 2,8-dibromo isomers), while others are instead tri-brominated, with an extra $\mathrm{Br}$ atom covalently bound to a carbon on the side of the molecule $\left(1,3,9-\mathrm{Br}_{3} \mathrm{PXX}\right)$. The intermolecular interactions controlling the assembly of phase 2 are mostly $\mathrm{XB}$, with some possible contribution from non-classical $\mathrm{O} \cdots \mathrm{H}-\mathrm{C}$ hydrogen bonds and secondary electrostatic $\mathrm{Br} \cdots \mathrm{H}$ interactions (Supplementary Fig. 2). Although all the analytical spectroscopic characterisations suggested that the $3,9-\mathrm{Br}_{2} \mathrm{PXX}$ obtained was pure (Supplementary Figs. 11-14), small traces of dibromo isomers and tribromo derivatives might still have been present in the sample due to their low solubility (see experimental procedures in the Supplementary Methods). The fact that these impurities appear in our STM study at a relatively high concentration may be due to a higher rate of sublimation than $3,9-\mathrm{Br}_{2} \mathrm{PXX}$, resulting in an overrepresentation when deposited onto the surface. In any case, the ability of clearly identifying these molecules by 

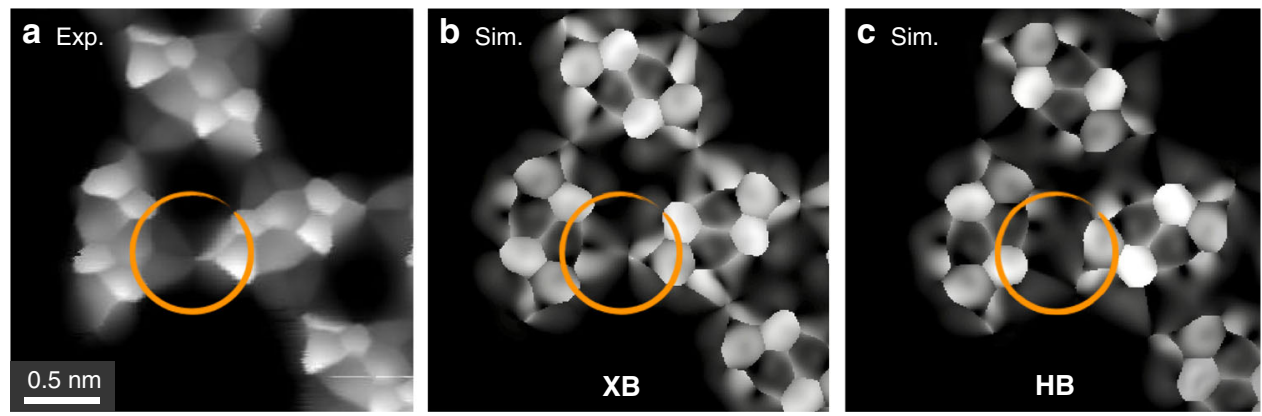

Fig. 5 Comparison between experimental and simulated HR-STM images of the 3,9-Br ${ }_{2} \mathbf{P X X}$ kagome assembly. a Constant height HR-STM image (same region as Fig. 4c). b Simulated HR-STM image (see text) of the XB assembly. c Simulated HR-STM image of the HB assembly. The orange circles highlight the $\mathrm{O} \cdots \mathrm{Br} / \mathrm{H}$ region.
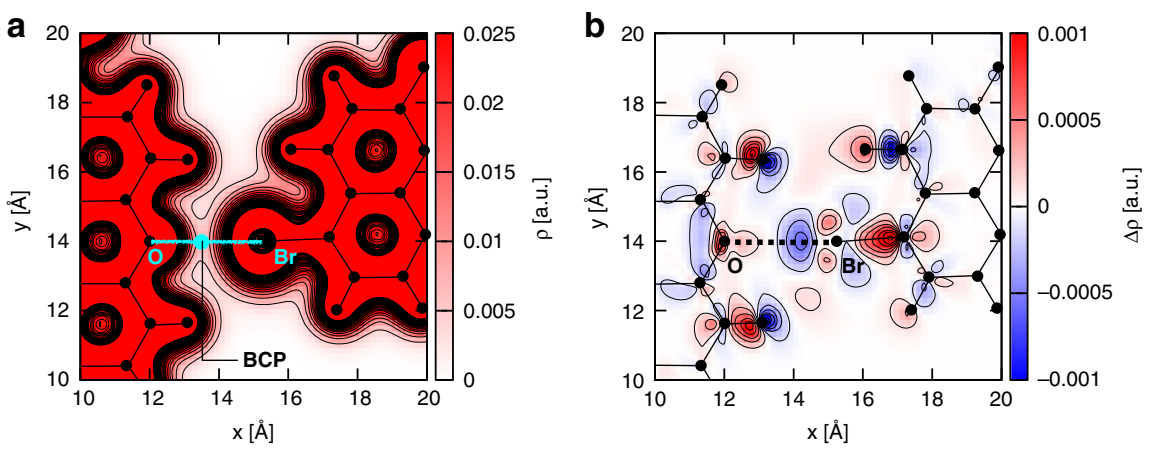

Fig. 6 Analysis of the electron density topology. a Contour map of the electron density $\rho$ projected onto the molecular plane $x y$. The location of the $O$ and $\mathrm{Br}$ atoms is explicitly shown, together with that of the bond critical point, BCP. $\mathbf{b}$ Electron density difference $\Delta \rho$, calculated as the difference between the electron density of the dimer and that of the two monomers.

HR-STM when other, conventional spectroscopic characterisation techniques fail to do so, is a further demonstration of the potential of sub-molecular resolution scanning probe microscopy as an analytical tool for chemical structure determination ${ }^{35}$. Interestingly, the minority phase 2 appears to be made exclusively of defective molecules (of various types), while the regular $3,9-\mathrm{Br}_{2} \mathrm{PXX}$ molecules all assemble into the kagome phase 1 structures.

Finally, HR-STM also shows that the compact phase 3 that develops at a higher molecular coverage is kept together by synergistic $\mathrm{HB}$ and XB (Supplementary Fig. 3). The molecules are arranged into parallel rows and interact with each other via nonclassical $\mathrm{O} \cdots \mathrm{H}-\mathrm{C}$ hydrogen bonds as well as type I halogen bonds between terminal $\mathrm{C}-\mathrm{Br}$ groups. Interestingly, this assembly is identical to that characterising the $3 \mathrm{D}$ crystalline phase of 3,9$\mathrm{Br}_{2} \mathrm{PXX}$ as determined by $\mathrm{X}$-ray diffraction (Supplementary Fig. 4), with one of the lattice parameters being slightly larger, most probably due to the molecule-surface interaction. Phase 3 is almost exclusively composed of regular 3,9- $\mathrm{Br}_{2} \mathrm{PXX}$ molecules, although occasional defective molecules (both $2,9-\mathrm{Br}_{2} \mathrm{PXX}$ and $2,8-\mathrm{Br}_{2} \mathrm{PXX}$ dibromo isomers and $1,3,9-\mathrm{Br}_{3} \mathrm{PXX}$ tribromo derivatives) are observed within the compact islands, and partially de-brominated molecules are sometimes found at the edges (Supplementary Fig. 5).

In conclusion, high-resolution STM performed with a COfunctionalised tip is found to be necessary to conclusively identify the arrangement of dibrominated $\mathrm{sp}^{2}$-hybridised molecules within an on-surface self-assembled molecular network. The experimental information provided by HRSTM provides the basis for a theoretical structural, electrostatic, and electron density topology analysis, allowing us to identify with great accuracy the emergence of halogen bonding as the intermolecular interaction that stabilises the observed molecular structures. Whereas standard metallic-tip STM is unable to differentiate between halogen and hydrogen assembly motifs, HR-STM clearly identifies Br...O halogen bonding as the only source of the observed kagome assemblies of 3,9$\mathrm{Br}_{2} \mathrm{PXX}$ on $\mathrm{Au}(111)$. DFT calculations support this result by predicting a higher stability of halogen versus hydrogen intermolecular binding while simulations of the HR-STM images demonstrate a remarkable agreement with the experimental data only for the halogen bonding interaction. HR-STM also identified the presence of defective molecules that escaped the scrutiny of traditional analytical methods and thereby fully solved the experimentally observed 2D self-assembly. Building upon the cases in which HR-SPM has been shown to be an invaluable tool for identifying the chemical structure of unknown molecules ${ }^{35-37}$ or for determining the intermediates and products of on-surface reactions ${ }^{38-40}$, this work exemplifies the unique analytical insight that HR-SPM can give in establishing the nanoscale arrangement of molecules within a supramolecular structure and the nature of the intermolecular interactions ruling the self-assembly. We believe that this methodology can be effectively used when investigating $2 \mathrm{D}$ networks originating from the self-assembly of planar molecules on surfaces and that a significant fraction of difficult or controversial molecular structures that have been discussed in the literature over the last decades could be quickly and clearly solved by using this approach.

\section{Methods}

Scanning tunnelling microscopy measurements. STM experiments were performed on a low temperature STM under ultra-high vacuum (UHV) conditions. The $\mathrm{Au}(111)$ single crystal was cleaned with multiple cycles of $\mathrm{Ar}^{+}$sputtering and annealing. 3,9- $\mathrm{Br}_{2} \mathrm{PXX}$ was deposited via sublimation $(483 \mathrm{~K})$ onto the $\mathrm{Au}(111)$ crystal, held at room temperature. The crystal was then cooled to $77 \mathrm{~K}$ or $7 \mathrm{~K}$ for 
STM analysis. Standard STM measurements were carried out with bias voltages ( $V$, applied to the sample) in the range of $\pm 2.0 \mathrm{~V}$ and tunnelling currents of $50-200$ pA. In order to perform high-resolution STM experiments, $\mathrm{CO}$ was leaked into the UHV system, adsorbed onto the $\mathrm{Au}(111)$ crystal held at $\sim 10 \mathrm{~K}$, and picked up by the STM tip. High-resolution STM images were taken in constant height mode (current channel). STM image analysis was performed with WSxM ${ }^{65}$, Gwyddion ${ }^{66}$ and LMAPper ${ }^{6}$.

Density Functional theory calculations. DFT calculations were performed using the mixed Gaussian and Plane-Waves (GPW) method implemented in the CP2K package ${ }^{68}$. As the description of both halogen bonding (XB) and hydrogen bonding (HB) interactions is known to be quite sensitive to the choice of the exchangecorrelation (XC) functional ${ }^{56}$, we have used two different fully self-consistent nonlocal XC functionals, namely vdW-DF ${ }^{69}$ and optB88-vdW ${ }^{70}$, to assess the reliability of our results. Goedecker-type pseudopotentials ${ }^{71}$ with four, one, six and seven valence electrons for $\mathrm{C}, \mathrm{H}, \mathrm{O}$ and $\mathrm{Br}$ respectively have been employed. The KohnSham orbitals were expanded in a Double-Zeta Valence plus Polarisation (DZVP) Gaussian-type basis set, while the plane wave cutoff for the finest level of the multigrid $^{68}$ has been set to $400 \mathrm{Ry}$ to efficiently solve the Poisson equation within periodic boundary conditions using the Quickstep scheme ${ }^{68}$. Brillouin zone integration was restricted to the supercell $\Gamma$-point. We have found that considering a single unit cell (in-plane dimensions of $\sim 20 \AA$ and containing three $3,9-\mathrm{Br}_{2} \mathrm{PXX}$ molecules, thus totalling 96 atoms), together with a vacuum region of $\sim 20 \AA$ inserted between the periodic replica of the 2D self-assemblies (along the direction normal to the assemblies planes) is sufficient to ensure an accuracy of the resulting total energy of $3 \mathrm{meV} / \mathrm{atom}$.

Image simulations. The simulated HR-STM images have been obtained thanks to the PP-AFM/STM framework of Hapala et al. ${ }^{64}$ and Krejčí et al. ${ }^{61}$. The COfunctionalized tip employed experimentally is approximated by a probe particle bonded to the STM tip: this bond is $4 \AA$ long and characterised by a lateral stiffness of $0.5 \mathrm{~N} / \mathrm{m}$. The charge of the probe particle is set equal to zero. As the experimental HR-STM images were obtained in constant height using very low bias voltages, all HR-STM simulated images were calculated as constant height dI/dV maps at the energy of the 3,9- $\mathrm{Br}_{2} \mathrm{PXX}$ highest occupied molecular orbital (HOMO). In order to describe the tunnelling process, we have considered the $s$ and $p$ orbitals of the sample and the $p_{x}$ and $p_{y}$ orbitals of the functionalized tip. The LennardJones and electrostatic fields characterising the sample have been obtained using simple point-charge electrostatics ${ }^{64}$ and the equilibrium configuration of the system was used, as obtained via the DFT calculations described above. The electronic density of states and the molecular orbitals of the sample have also been obtained via the $\mathrm{CP} 2 \mathrm{~K}$ code.

Further details on the STM methods as well as the full synthetic details are given in the Supplementary Methods.

\section{Data availability}

All data needed to evaluate the conclusions in the paper are present in the paper and/or the Supplementary Information. Crystallographic data (excluding structure factors) for compound 3,9- $\mathrm{Br}_{2} \mathrm{PXX}$ reported in this paper have been deposited at the Cambridge Crystallographic Data Centre, under deposition number 1901257. These data can be obtained free of charge from The Cambridge Crystallographic Data Centre via www.ccdc. cam.ac.uk/data_request/cif. Additional data supporting the findings of this study are available from the corresponding author upon reasonable request.

\section{Code availability}

The electronic structure calculations presented in this work have been obtained by the CP2K (version 4.1) package, which is an open source code freely available at https:// www.cp2k.org. The simulated HR-STM images have been computed via the PPSTM framework of Ondřej Krejčí, which is freely available via GitHub at https://github.com/ ondrejkrejci/PPSTM.

Received: 9 October 2019; Accepted: 24 March 2020;

Published online: 30 April 2020

\section{References}

1. Gilday, L. C. et al. Halogen bonding in supramolecular chemistry. Chem. Rev. 115, 7118-7195 (2015)

2. Parthasarathi, R. et al. Hydrogen bonding - new insights (Springer, 2006).

3. Slater, A. G., Perdigão, L. M. A., Beton, P. H. \& Champness, N. R. Surfacebased supramolecular chemistry using hydrogen bonds. Acc. Chem. Res. 47, 3417-3427 (2014).

4. Otero, R., Gallego, J. M., de Parga, A. L. V., Martín, N. \& Miranda, R. Molecular self assembly at solid surfaces. Adv. Mater. 23, 5148-5176 (2011).
5. Barth, J. V. Molecular architectonic on metal surfaces. Annu. Rev. Phys. Chem 58, 375-407 (2007).

6. Bonifazi, D., Mohnani, S. \& Llanes-Pallas, A. Supramolecular chemistry at interfaces: molecular recognition on nanopatterned porous surfaces. Chem. Eur. J. 15, 7004-7025 (2009).

7. Beale, T. M., Chudzinski, M. G., Sarwar, M. G. \& Taylor, M. S. Halogen bonding in solution: thermodynamics and applications. Chem. Soc. Rev. 42, 1667-1680 (2013)

8. Metrangolo, P., Meyer, F., Pilati, T., Resnati, G. \& Terraneo, G. Halogen bonding in supramolecular chemistry. Angew. Chem. Int. Ed. 47, 6114-6127 (2008).

9. Erdélyi, M. Halogen bonding in solution. Chem. Soc. Rev. 41, 3547-3557 (2012).

10. Clarke, S. M. et al. Observation of a two-dimensional halogen-bonded cocrystal at sub-monolayer coverage using synchrotron X-ray diffraction. Chem. Commun. 47, 2526-2528 (2011).

11. Silly, F. Concentration-dependent two-dimensional halogen-bonded selfassembly of 1,3,5-tris(4-iodophenyl)benzene molecules at the solid-liquid interface. J. Phys. Chem. C 121, 10413-10418 (2017).

12. Sacchi, M. et al. Combined diffraction and density functional theory calculations of halogen-bonded cocrystal monolayers. Langmuir 29, 14903-14911 (2013).

13. Mukherjee, A., Teyssandier, J., Hennrich, G., De Feyter, S. \& Mali, K. S. Twodimensional crystal engineering using halogen and hydrogen bonds: towards structural landscapes. Chem. Sci. 8, 3759-3769 (2017).

14. Zheng, Q.-N. et al. Formation of halogen bond-based 2D supramolecular assemblies by electric manipulation. J. Am. Chem. Soc. 137, 6128-6131 (2015).

15. Jeon, U. S. et al. Two-dimensional networks of brominated Y-shaped molecules on Au(111). Appl. Surf. Sci. 432, 332-336 (2018).

16. Jang, W. J. et al. Tetragonal porous networks made by rod-like molecules on $\mathrm{Au}\left(\begin{array}{lll}1 & 1 & 1\end{array}\right)$ with halogen bonds. Appl. Surf. Sci. 309, 74-78 (2014).

17. Yoon, J. K. et al. Visualizing halogen bonds in planar supramolecular systems. J. Phys. Chem. C 115, 2297-2301 (2011)

18. Song, W., Martsinovich, N., Heckl, W. M. \& Lackinger, M. Thermodynamics of halogen bonded monolayer self-assembly at the liquid-solid interface. Chem. Commun. 50, 13465-13468 (2014).

19. Brewer, A. Y. et al. Supramolecular self-assembled network formation containing $\mathrm{N} \cdots \mathrm{Br}$ halogen bonds in physisorbed overlayers. Phys. Chem. Chem. Phys. 16, 19608-19617 (2014).

20. Noh, S. K. et al. Supramolecular Cl $\cdots \mathrm{H}$ and $\mathrm{O} \cdots \mathrm{H}$ interactions in selfassembled 1,5-dichloroanthraquinone layers on $\mathrm{Au}(111)$. ChemPhysChem 14, 1177-1181 (2013)

21. Kawai, S. et al. Extended halogen bonding between fully fluorinated aromatic molecules. ACS Nano 9, 2574-2583 (2015).

22. Han, Z. et al. Imaging the halogen bond in self-assembled halogenbenzenes on silver. Science 358, 206-210 (2017).

23. Huang, H. et al. Competition between hexagonal and tetragonal hexabromobenzene packing on $\mathrm{Au}(111)$. ACS Nano 10, 3198-3205 (2016).

24. Walch, H., Gutzler, R., Sirtl, T., Eder, G. \& Lackinger, M. Material- and orientation-dependent reactivity for heterogeneously catalyzed carbonbromine bond homolysis. J. Phys. Chem. C 114, 12604-12609 (2010).

25. Yasuda, S., Furuya, A. \& Murakoshi, K. Control of a two-dimensional molecular structure by cooperative halogen and hydrogen bonds. RSC Adv. 4, 58567-58572 (2014)

26. Guo, Z. et al. Role of halogen $\cdots$ halogen interactions in the $2 \mathrm{D}$ crystallization of n-semiconductors at the liquid-solid interface. Phys. Chem. Chem. Phys. 19, 31540-31544 (2017)

27. Chen, Q. et al. Two-dimensional OPV4 self-assembly and its coadsorption with alkyl bromide: from helix to lamellar. Chem. Commun., 3765-3767 (2009).

28. Temirov, R., Soubatch, S., Neucheva, O., Lassise, A. C. \& Tautz, F. S. A novel method achieving ultra-high geometrical resolution in scanning tunnelling microscopy. N. J. Phys. 10, 053012 (2008).

29. Gross, L. et al. The chemical structure of a molecule resolved by atomic force microscopy. Science 325, 1110-1114 (2009)

30. Weiss, C. et al. Imaging pauli repulsion in scanning tunneling microscopy. Phys. Rev. Lett. 105, 2-5 (2010).

31. Kichin, G., Weiss, C., Wagner, C., Tautz, F. S. \& Temirov, R. Single molecule and single atom sensors for atomic resolution imaging of chemically complex surfaces. J. Am. Chem. Soc. 133, 16847-16851 (2011).

32. Chiang, C. L., Xu, C., Han, Z. \& Ho, W. Real-space imaging of molecular structure and chemical bonding by single-molecule inelastic tunneling probe. Science 344, 885-888 (2014)

33. Mohn, F., Schuler, B., Gross, L. \& Meyer, G. Different tips for high-resolution atomic force microscopy and scanning tunneling microscopy of single molecules. Appl. Phys. Lett. 102, 073109 (2013). 
34. Mönig, H. et al. Quantitative assessment of intermolecular interactions by atomic force microscopy imaging using copper oxide tips. Nat. Nanotechnol. 13, 371-375 (2018).

35. Gross, L. et al. Organic structure determination using atomic-resolution scanning probe microscopy. Nat. Chem. 2, 821-825 (2010).

36. Hanssen, K. O. et al. A combined atomic force microscopy and computational approach for the structural elucidation of breitfussin A and B: Highly modified halogenated dipeptides from thuiaria breitfussi. Angew. Chem. Int Ed. 51, 12238-12241 (2012).

37. Schuler, B., Meyer, G., Peña, D., Mullins, O. C. \& Gross, L. Unraveling the molecular structures of asphaltenes by atomic force microscopy. J. Am. Chem. Soc. 137, 9870-9876 (2015).

38. Riss, A. et al. Imaging single-molecule reaction intermediates stabilized by surface dissipation and entropy. Nat. Chem. 8, 678-683 (2016).

39. Oteyza, D. G. D. et al. Direct imaging of covalent bond structure in singlemolecule chemical reactions. Science 340, 1434-1437 (2013).

40. Riss, A. et al. Local electronic and chemical structure of oligo-acetylene derivatives formed through radical cyclizations at a surface. Nano Lett. 14, 2251-2255 (2014).

41. Hellerstedt, J. et al. Aromatic azide transformation on the $\mathrm{Ag}(111)$ surface studied by scanning probe microscopy. Angew. Chem. Int. Ed. 58, 2266-2271 (2019).

42. Hieulle, J. et al. On-surface route for producing planar nanographenes with azulene moieties. Nano Lett. 18, 418-423 (2018).

43. Nguyen, G. D. et al. Atomically precise graphene nanoribbon heterojunctions from a single molecular precursor. Nat. Nanotechnol. 12, 1077-1082 (2017).

44. Sánchez-Sánchez, C. et al. Purely armchair or partially chiral: noncontact atomic force microscopy characterization of dibromo-bianthryl-based graphene nanoribbons grown on $\mathrm{Cu}(111)$. ACS Nano 10, 8006-8011 (2016).

45. Liu, M. et al. Graphene-like nanoribbons periodically embedded with fourand eight-membered rings. Nat. Commun. 8, 14924 (2017).

46. Weiss, C., Wagner, C., Temirov, R. \& Tautz, F. S. Direct imaging of intermolecular bonds in scanning tunneling microscopy. J. Am. Chem. Soc. 132, 11864-11865 (2010).

47. Sweetman, A. M. et al. Mapping the force field of a hydrogen-bonded assembly. Nat. Commun. 5, 1-7 (2014).

48. Hämäläinen, S. K. et al. Intermolecular contrast in atomic force microscopy images without intermolecular bonds. Phys. Rev. Lett. 113, 186102 (2014).

49. Jarvis, S. P. Resolving intra- and inter-molecular structure with non-contact atomic force microscopy. Int. J. Mol. Sci. 16, 19936-19959 (2015).

50. Fermi, A. et al. Tailoring colors by $\mathrm{O}$ annulation of polycyclic aromatic hydrocarbons. Chem. Eur. J. 23, 2363-2378 (2017).

51. Stassen, D., Demitri, N. \& Bonifazi, D. Extended O-doped polycyclic aromatic hydrocarbons. Angew. Chem. Int. Ed. 55, 5947-5951 (2016).

52. Sciutto, A. et al. Customizing photoredox properties of PXX-based dyes through energy level rigid shifts of frontier molecular orbitals. Chem. Eur. J. 24, 4382-4389 (2018).

53. Berezin, A., Biot, N., Battisti, T. \& Bonifazi, D. Oxygen-doped zig-zag molecular ribbons. Angew. Chem. Int. Ed. 57, 8942-8946 (2018).

54. Kobayashi, N., Sasaki, M. \& Nomoto, K. Stable peri-xanthenoxanthene thin-film transistors with efficient carrier injection. Chem. Mater. 21, 552-556 (2009).

55. Wang, L., Duan, G., Ji, Y. \& Zhang, H. Electronic and charge transport properties of peri-xanthenoxanthene: the effects of heteroatoms and phenyl substitutions. J. Phys. Chem. C. 116, 22679-22686 (2012).

56. Kolár, M. H. \& Hobza, P. Computer modeling of halogen bonds and other $\sigma$ hole interactions. Chem. Rev. 116, 5155-5187 (2016).

57. Driver, S. M., Zhang, T. \& King, D. A. Massively cooperative adsorbateinduced surface restructuring and nanocluster formation. Angew. Chem. Int. Ed. 46, 700-703 (2007).

58. Rossel, F., Brodard, P., Patthey, F., Richardson, N. V. \& Schneider, W.-D Modified herringbone reconstruction on $\mathrm{Au}(111)$ induced by self-assembled Azure A islands. Surf. Sci. 602, L115-L117 (2008).

59. Kutsenko, V. Y. et al. Alkylthiol self-assembled monolayers on $\mathrm{Au}(111)$ with tailored tail groups for attaching gold nanoparticles. Nanotechnology $\mathbf{2 8}$, 235603 (2017)

60. Faraggi, M. N. et al. Bonding and charge transfer in metal-organic coordination networks on $\mathrm{Au}(111)$ with strong acceptor molecules. J. Phys. Chem. C. 116, 24558-24565 (2012).

61. Krejčí, O., Hapala, P., Ondráček, M. \& Jelínek, P. Principles and simulations of high-resolution STM imaging with a flexible tip apex. Phys. Rev. B 95, 045407 (2017).

62. Bondi, A. Van der waals volumes and radii. J. Phys. Chem. 68, 441-451 (1964).

63. Desiraju, G. R. et al. Definition of the halogen bond (IUPAC Recommendations 2013). 85, 1711-1713 (2013).

64. Hapala, P. et al. Mechanism of high-resolution STM/AFM imaging with functionalized tips. Phys. Rev. B 90, 085421 (2014).
65. Horcas, I. et al. WSXM: a software for scanning probe microscopy and a tool for nanotechnology. Rev. Sci. Instrum. 78, 013705 (2007).

66. Nečas, D. \& Klapetek, P. Gwyddion: an open-source software for SPM data analysis. Open Phys. 10, 181-188 (2012).

67. Perdigão, L. M. A. LMAPper - The SPM and Mol Viewer, https://sourceforge net/projects/spm-and-mol-viewer/.

68. VandeVondele, J. et al. Quickstep: Fast and accurate density functional calculations using a mixed Gaussian and plane waves approach. Comput. Phys. Commun. 167, 103-128 (2005).

69. Dion, M., Rydberg, H., Schroder, E., Langreth, D. C. \& Lundqvist, B. I. Van der Waals density functional for general geometries. Phys. Rev. Lett. 92, 246401 (2004)

70. Klimes, J., Bowler, D. R. \& Michaelides, A. Chemical accuracy for the van der Waals density functional. J. Phys. Condens. Matter 22, 022201 (2010).

71. Goedecker, S., Teter, M. \& Hutter, J. J. P. R. B. Separable dual-space Gaussian pseudopotentials. Phys. Rev. B 54, 1703 (1996).

\section{Acknowledgements}

G.C. acknowledges financial support from the University of Warwick and from the EU through the ERC Grant "VISUAL-MS" (Project ID: 308115). D.B. gratefully acknowledges the EU through the MSCA-RISE project "INFUSION" (Project ID: 734834), the ERC Grant "COLORLANDS" (Project ID: 280183), and Cardiff University. We thank Dr. Benson Kariuki, Deborah Romito and Nicolas Biot for the X-ray analysis, Dr. Rober L. Jenkins for the help with NMR experiments, Thomas Williams for his support with MALDI-HRMS analysis, and the Analytical Service at the School of Chemistry, Cardiff University. G.C.S. is grateful to the Centre for Scientific Computing at the University of Warwick for providing computational resources. G.C.S. also acknowledges the use of Athena at HPC Midlands+ (funded by the EPSRC on grant EP/P020232/) in this research, as part of the HPC Midlands+ consortium.

\section{Author contributions}

J.L. performed the surface deposition experiments, the STM and HR-STM experiments, analysed the experimental data, and wrote the first draft of the manuscript. H.P. performed DFT calculations. G.C.S. performed the electronic structure calculations, computed the simulated HR-STM images and analysed the results. L.D. and D.B. designed synthesised, and characterised the molecular module. G.C. conceived and coordinated the research project. All authors participated in discussing the data and editing the manuscript.

\section{Competing interests}

The authors declare no competing interests.

\section{Additional information}

Supplementary information is available for this paper at https://doi.org/10.1038/s41467 020-15898-2.

Correspondence and requests for materials should be addressed to G.C.S., D.B. or G.C

Peer review information Nature Communications thanks Philip Moriarty and the other anonymous, reviewer(s) for their contribution to the peer review of this work. Peer reviewer reports are available.

Reprints and permission information is available at http://www.nature.com/reprints

Publisher's note Springer Nature remains neutral with regard to jurisdictional claims in published maps and institutional affiliations.

Open Access This article is licensed under a Creative Commons Attribution 4.0 International License, which permits use, sharing, adaptation, distribution and reproduction in any medium or format, as long as you give appropriate credit to the original author(s) and the source, provide a link to the Creative Commons license, and indicate if changes were made. The images or other third party material in this article are included in the article's Creative Commons license, unless indicated otherwise in a credit line to the material. If material is not included in the article's Creative Commons license and your intended use is not permitted by statutory regulation or exceeds the permitted use, you will need to obtain permission directly from the copyright holder. To view a copy of this license, visit http://creativecommons.org/ licenses/by/4.0/

(C) The Author(s) 2020 